(Se) former pour enseigner le français à ceux qui ne le parlent pas nativement -

\title{
Yoshiko SUGIYAMA. Sur les mêmes bancs d'école : Louis Machuel et la rencontre franco-arabe en Tunisie, lors du Protectorat français (1883-1908).
}

\section{Gérard Vigner}

\section{(2) OpenEdition}

\section{Journals}

Édition électronique

URL : https://journals.openedition.org/dhfles/4333

DOI : $10.4000 /$ dhfles.4333

ISSN : 2221-4038

Éditeur

Société Internationale pour l'Histoire du Français Langue Étrangère ou Seconde

Édition imprimée

Date de publication : 1 décembre 2015

Pagination : 195-200

ISSN : 0992-7654

Référence électronique

Gérard Vigner, "Yoshiko SUGIYAMA. Sur les mêmes bancs d'école : Louis Machuel et la rencontre franco-arabe en Tunisie, lors du Protectorat français (1883-1908). », Documents pour l'histoire du français langue étrangère ou seconde [En ligne], 55 | 2015, mis en ligne le 19 novembre 2017, consulté le 28 mars 2023. URL : http://journals.openedition.org/dhfles/4333 ; DOI : https://doi.org/10.4000/ dhfles. 4333

Ce document a été généré automatiquement le 28 mars 2023

Tous droits réservés 


\title{
Yoshiko SUGIYAMA. Sur les mêmes bancs d'école : Louis Machuel et la rencontre franco-arabe en Tunisie, lors du Protectorat français (1883-1908).
}

\author{
Gérard Vigner
}

1 Atelier national de reproduction des thèses, Lille, Thèses à la carte, $454 \mathrm{p}$. ISBN : 978-2-7295-7194-8

2 Lorsque les autorités françaises décident d'ouvrir dans les colonies des écoles destinées à scolariser les enfants indigènes, elles le font en déléguant ce pouvoir aux gouverneurs en poste dans les territoires placés sous leur autorité. Le ministère de l'Instruction publique, dans la période qui nous intéresse ici, en gros entre 1880 et 1914, ne dispose en effet d'aucune autorité sur ces nouveaux réseaux d'écoles qui pour leur fonctionnement émargeront au budget de la colonie au titre des dépenses civiles. Peu familiers du domaine, les gouverneurs vont, pour organiser ce réseau et en définir les programmes ainsi que les conditions de recrutement et de nomination d'un personnel spécifique, soit enseignants métropolitains détachés, soit enseignants indigènes formés localement, nommer un directeur de l'enseignement. Personne dotée d'un pouvoir d'initiative important, supérieur dans bien des cas à celui d'un recteur d'académie dans la France métropolitaine de l'époque, le directeur de l'enseignement, tout en s'inspirant des programmes français, va créer et faire fonctionner un système éducatif original, adapté au territoire et à ses populations.

3 La personnalité du directeur, notamment dans les phases initiales de mise en place, va jouer un rôle particulièrement important. Selon sa sensibilité, la formation de départ qui est la sienne, son expérience professionnelle antérieure et le contexte politique propre au territoire aussi, le nouveau directeur va orienter cette école dans une direction qui peut correspondre à l'idée qu'il se fait d'une école au service du projet 
civilisateur de la colonisation. À ce titre, Louis Machuel (1848-1922), premier directeur de l'enseignement public en Tunisie, de 1883 à 1908, mérite une attention toute particulière pour l'originalité de sa position, notamment vis-à-vis de l'enseignement de la langue arabe et la durée de sa mission - il resta vingt-cinq années en poste. Responsable au plus haut niveau de la politique éducative de la France dans la Tunisie nouvellement conquise (traité du Bardo, 12 mai 1881), il aurait pu s'en tenir à une politique soucieuse de diffuser exclusivement la langue française, faisant de l'école un autre instrument de la domination coloniale. Or, sans remettre en question une telle orientation, ce qui va d'ailleurs le placer au cœur de contradictions difficilement surmontables, il va s'efforcer de défendre un enseignement de la langue arabe qui pourrait s'adresser à l'ensemble des élèves scolarisés, qu'ils soient d'origine européenne ou tunisienne.

La thèse dont nous rendons compte ici, déjà ancienne et soutenue en 2007, mérite cependant d'être signalée, car elle permet de situer la personnalité et les pensées d'un arabisant, venu d'Algérie où son père exerçait comme instituteur arabisant et interprète de l'armée française. Ce dernier lui fera fréquenter, parallèlement à l'école française, une école coranique, situation peu commune dans cette Algérie annexée par la France et dans laquelle la population européenne se tenait à la plus grand distance possible d'une population arabe à la fois redoutée et méprisée. Yoshisko Sugiyama consacre environ une vingtaine de pages à la restitution de la vie de Louis Machuel, détails précieux qui éclairent le personnage, mais que l'on aurait aimés peut-être plus développés, notamment pour ce qui est de la dimension publique ou semi-publique de sa vie. S'il est en effet nommé directeur de l'enseignement public en Tunisie, par Jules Ferry, ministre de l'Instruction publique, en 1883, il le doit aussi aux nombreux appuis dont il put bénéficier auprès du personnel politique et éducatif de la III $^{e}$ République commençante. Machuel bénéficie en effet de l'appui de Ferdinand Buisson, alors directeur de l'Enseignement primaire qui le remarque lors d'une visite d'inspection en Algérie, et qui lui confie alors une visite d'inspection en Tunisie. Il le recommandera auprès de Paul Cambon, premier Résident général en Tunisie, qui lui proposera de devenir directeur de l'enseignement public. Il est présent à Paris le 21 juillet 1883, lors de la séance fondatrice de l'Alliance française. Mais il est intéressant de relever ici que Louis Machuel est remarqué d'abord pour sa connaissance du monde et de la langue arabes, atouts considérés comme essentiels dans une Tunisie où les autorités françaises veulent conduire une politique différente de celle adoptée en Algérie. Mais peu de détails en revanche sur les hostilités que put soulever sa politique, notamment dans le milieu des colons français de Tunisie, représentés par Victor de Carnières, leur porteparole le plus influent à la tête de son journal La Tunisie française, et qui ne manquera pas, à plusieurs reprises, de critiquer sa politique. Les difficultés rencontrées dans sa mission sont d'ailleurs évoquées dans l'article Tunisie, signé de Benjamin Buisson dans Le Nouveau dictionnaire de pédagogie et d'enseignement primaire (édition de 1911), et l'arrivée de son successeur, Sébastien Charléty ${ }^{1}$, nommé en Tunisie comme inspecteur général de l'enseignement professionnel des indigènes, est saluée en termes élogieux, critique à peine masquée de l'œuvre de Louis Machuel. Observons au passage que Charléty lui-même n'était pas un arabisant, ce qui témoignait d'un changement d'orientation de la politique de la Résidence. Louis Machuel démissionne, est admis à faire valoir des droits à la retraite et restera en Tunisie jusqu'à sa mort, en $1922^{2}$.

Mais l'essentiel de la thèse est consacré à l'analyse de la pensée de Louis Machuel et de ses politiques scolaires dans un univers social et culturel particulièrement complexe. 
En effet, Louis Machuel trouve en arrivant à côté des Tunisiens, une population italienne et maltaise déjà bien implantée, numériquement plus nombreuse que la population d'origine française, et qui parle couramment l'arabe, et une population juive dont les enfants commencent à être scolarisés dans les écoles de l'Alliance israélite universelle. La première école de l'AIU est ouverte à Tunis en 1878.

6 Une première partie décrit l'environnement dans lequel va prendre place la politique éducative de la France. Yoshiko Sugiyama montre bien notamment combien tous ceux qui veillent à la mise en place du Protectorat (Paul Cambon, Jules Ferry, le cardinal Lavigerie, Jean-Jules Jusserand) ne veulent pas que soient reproduites les erreurs commises en Algérie en matière de conquête et de domination. La place et le rôle de l'Alliance française dans son appui aux politiques de scolarisation y sont clairement exposés.

7 La suite de la thèse, dans un parcours de lecture assez complexe, met l'accent sur les idéaux qui habitent Machuel au contact des nombreux acteurs culturels présents dans la Tunisie de l'époque. On a en effet parfois du mal à se repérer dans une chronologie, pourtant essentielle, dans la mesure où les positions de la Résidence et des milieux tunisiens évolueront tout au long de l'administration de Machuel. Mais il est important de découvrir le très riche terreau d'idées et de projets que portent tous ceux qui en Tunisie sont intéressés par une Tunisie moderne. Yoshiko Sugiyama en entreprend une étude détaillée qui montre la diversité des sensibilités, des projets, et la difficulté pour Louis Machuel à organiser et faire évoluer sa politique scolaire dans un univers aux sensibilités et aux intérêts si divergents.

8 L'action de Louis Machuel en direction de l'école est abordée sous l'angle du choix des langues à enseigner. S'il n'existe pas en Tunisie d'école indigène, comme en Algérie par exemple, on y ouvre bien des écoles primaires franco-arabes dans lequel sont scolarisés les enfants essentiellement tunisiens, avec un enseignement de l'arabe et du français, ce qui correspondait au rêve de Louis Machuel de voir les élèves de toutes origines partager "les mêmes bancs d'école ", mais à côté des écoles européennes, accueillant les enfants français et italiens. Ce rêve ne put cependant se réaliser. Peut-être eût-il été utile, pour mieux éclairer le lecteur, de préciser ce que furent les programmes et les horaires, modifiés à de nombreuses reprises, témoignant des incertitudes de la politique éducative de la Résidence en la matière. Yoshiko Sugiyama évoque à ce sujet Louis Machuel auteur de manuels de langage à destination des enfants des écoles européennes et franco-arabes, à côté des manuels produits par Irénée Carré. Si les deux démarches se rapprochent dans une même logique qui consiste à faire de la leçon de langage " une leçon de choses ", mots et objets étant régulièrement associés, Machuel se distingue en revanche de Carré par le souci qui est le sien de prendre appui sur la langue maternelle des élèves, l'arabe et l'italien en l'occurrence. Louis Machuel étendra par la suite l'usage de ses manuels à l'ensemble des publics des élèves des colonies avec la Méthode de lecture et de langage à destination des élèves indigènes et étrangers des colonies françaises, publiée chez Armand Colin.

9 La politique de laïcisation est décrite dans les particularités de sa mise en œuvre, non seulement à l'égard de la place de l'islam dans les écoles franco-arabes et de la tradition hébraïque dans les écoles juives, mais aussi à l'égard des écoles congréganistes, nombreuses, bien implantées. Le cardinal Lavigerie est là, ardent soutien de la langue française et des écoles catholiques, qui ne peut qu'être ménagé. En face, la francmaçonnerie de Tunisie, tout aussi influente et organisée, avec ses nombreux contacts à 
Paris, ne manque pas de critiquer les décisions de Louis Machuel en la matière, raisonnables pourtant, en ce sens qu'aucun établissement ne sera fermé. Louis Machuel s'efforce dans les établissements publics de faire prévaloir le principe de la neutralité laïque, ce qui correspondait, semble-t-il, à sa position personnelle par rapport à la religion.

Nombreux furent aussi les projets de Machuel en direction d'un islam universitaire et académique. Dans une Tunisie où vivent de nombreux réformateurs tunisiens nourris par les pensées réformatrices venues d'Égypte, Machuel souhaite, en accord avec la Résidence, René Millet le Résident de l'époque lui apportant son appui, faire de la Grande Mosquée de la Zaytûna une concurrente de Al Azhar au Caire. La Khaldûniyya est fondée en 1896, en accord avec les réformateurs tunisiens, et poursuivra son activité jusqu'en 1958, deux années après l'indépendance. Destinée à favoriser un islam universitaire, cette association vise à moderniser la culture classique islamique. Les pages consacrées aux relations de Louis Machuel avec les institutions de culture et de langue arabes, comme avec les milieux intellectuels tunisiens, sont particulièrement intéressantes, car elles éclairent un pan de l'action de Louis Machuel, ordinairement moins connu, celui de l'arabisant, tunisophile, pris dans ses contradictions d'homme d'ouverture, mais en même temps représentant de l'autorité coloniale, d'une France soucieuse de développer une politique arabe, ce qui ne correspondait pas forcément à l'idée que les Jeunes Tunisiens se faisaient de l'évolution de la Tunisie. Louis Machuel n'a pas su ou pas voulu percevoir ces aspirations.

11 Le travail de Yoshiko Sugiyama s'appuie sur un dépouillement minutieux de nombreuses sources d'archives, notamment celles des Archives nationales tunisiennes qui rendent compte des activités de la direction de l'Enseignement public de Tunisie et qui apportent de très nombreuses et de très riches informations sur le climat intellectuel et politique dans lequel prend place le parcours intellectuel de Louis Machuel.

Pour toutes celles et tous ceux qui s'intéressent à cet arabisant français dans une Afrique du nord sous domination coloniale, on renverra encore aux travaux de Noriyuki Nishiyama ${ }^{3}$, plus particulièrement consacrés au pédagogue que fut Louis Machuel et à ses choix se rapportant à l'enseignement du français. De même on lira avec profit l'étude d'Alain Messaoudi «Les arabisants français et la 'réforme' en Afrique du Nord. Autour de Louis Machuel (1848-1922) et de ses contemporains $»^{4}$ qui situe Louis Machuel et son œuvre dans un cadre plus large, celui des arabisants français qui appelaient de leurs vœux une réforme profonde des sociétés musulmanes d'Afrique du nord. Tradition réformatrice qui trouve son origine en Algérie et que Louis Machuel va s'efforcer de transporter en Tunisie avec les difficultés que l'on sait.

\section{NOTES}

1. Sébastien Charléty sera par la suite nommé recteur de l'académie de Strasbourg en 1919, puis recteur de l'académie de Paris en 1927. 
2. Louis Machuel ne quittera pas la Tunisie et y séjournera continument de 1883 à sa mort, hormis de brefs séjours en France, lors des vacances d'été.

3. "L'enseignement du français aux indigènes à la croisée des cultures politiques sous la III ${ }^{\mathrm{e}}$ République : comment la mutation de la politique coloniale s'est articulée avec la politique linguistique? », par Nishiyama Noriyuki, université de Niigata (Japon), Marges linguistiques, juillet 2004 ; Nishiyama Noriyuki, «La pédagogie bilingue de Louis Machuel et la politique du protectorat en Tunisie à la fin du XIX ${ }^{\mathrm{e}}$ siècle », Revue japonaise de didactique du français, vol. $1, \mathrm{n}^{\circ} 1$, 2006.

4. In Odile MOREAU (dir.) (2009). Réforme de l'État et réformismes au Maghreb (XIX ${ }^{e}-X X^{e}$ siècles), coll. Socio-anthropologie des mondes méditerranéens, Paris: IRMC-L'Harmattan, 368 p., ISBN: 978-2-296-11087-8. 